\title{
MEAN GROWTH OF INNER FUNCTIONS
}

\author{
DOMINGO A. HERRERO ${ }^{1}$
}

Abstract. Let $A$ be a closed subset of the closed unit disc. It is shown that there exists a "universal growth function" $\varphi(r, A)$ such that $1-\int_{0}^{2 \pi}\left|q\left(r e^{i x}\right)\right|^{2} d x / 2 \pi=O(\varphi(r, A))$ for all inner functions $q(z)$ whose zeroes lie in $A \cap\{|z|<1\}$ and whose singularities in the unit circle lie on $A \cap\{|z|=1\}$, if and only if the Lebesgue measure of $A \cap\{|z|=1\}$ is zero.

The $H^{2}$ Hardy class of functions in the unit disc $D=\{z:|z|<1\}$ of the complex plane is, by definition, the set of all functions $f(z)=\sum_{n=0}^{\infty} c_{n} z^{n}$, analytic on $D$, satisfying

$$
\lim \sup (r \rightarrow 1) M(f, r)<\infty,
$$

where $M(f, r)=\int_{0}^{2 \pi}\left|f\left(r e^{i x}\right)\right|^{2} d m=\sum_{n=0}^{\infty}\left|c_{n}\right|^{2} r^{2 n}, 0 \leqq r<1 \quad(d m=(1 / 2 \pi) d x$ is the normalized Lebesgue measure on $\partial D$ ).

It is clear that $M(f, r)$ is convex and nondecreasing with $r$ and that $M(f, r)$ converges to $M(f)=\sum_{n=0}^{\infty}\left|c_{n}\right|^{2}<\infty$, as $r \rightarrow 1$. However, by taking suitable lacunary series, it can be easily seen that there is no function $\varphi(r)$ such that

(1) $\varphi(r)$ is positive and decreasing for $r \in[0,1)$, and $\lim (r \rightarrow 1) \varphi(r)=0$, and $M(f)-M(f, r)=O(\varphi(r))$, for all $f \in H^{2}$.

The problem that we want to analyze here is this:

Is it possible to find a function $\varphi(r)$, satisfying (1) and such that

$$
1-M(q, r)=O(\varphi(r))
$$

holds for every inner function $q(z)$ ?

We recall that $q \in H^{2}$ is inner if it has radial limits $q\left(e^{i x}\right)=\lim (r \rightarrow 1) q\left(r e^{i x}\right)$ of modulus one a.e. $(\mathrm{dm})$ (for the existence of these limits and basic properties of $H^{2}$ functions and inner functions, see [2]).

Our first result provides an affirmative answer for a particular class of inner functions. The examples following the theorem will show that the

Received by the editors March 29, 1972 and, in revised form, May 25, 1972.

AMS (MOS) subject classifications (1970). Primary 30A76, 30A78.

Key words and phrases. Mean growth in $L^{2}$-norm, inner function, Blaschke product, singular part, singular measure, singularities in the boundary.

1 This research was partially supported by NSF grants GP-14255 and GU-3171. 
result is sharp; in particular, there is no function $\varphi(r)$ satisfying (1) such that (2) holds for every inner function. These results are contained in the author's thesis [1].

The canonical expression of an inner function $q(z)$ is (see [2]) $q(z)=$ $\lambda b(z) s(z)$, where $\lambda \in \partial D$,

$$
b(z)=\prod_{k}\left(\bar{a}_{k} /\left|a_{k}\right|\right) \cdot\left(a_{k}-z\right) /\left(1-\bar{a}_{k} z\right)(=\text { Blaschke product of } q)
$$

with $a_{k} \in D$ for all $k$ and $\sum_{k}\left(1-\left|a_{k}\right|\right)<\infty\left(\bar{a}_{k} /\left|a_{k}\right|=1\right.$, if $\left.a_{k}=0\right)$ and

$$
s(z)=e(z, \mu)=\exp \left\{-\int_{0}^{2 \pi}\left(e^{i t}+z\right) /\left(e^{i t}-z\right) d \mu(t)\right\}
$$

(=singular part of $q$ ), where $\mu$ is a finite nonnegative Borel measure on $\partial D$, singular with respect to $d m$. We shall define

$$
\operatorname{Sp}(q)=\operatorname{supp}(\mu) \cup \operatorname{closure}\left\{a_{k}\right\}
$$

( $q(z)$ can be continued analytically to $z=\alpha$, for some $\alpha$ in $\partial D$, if and only if $\alpha \notin \operatorname{Sp}(q))$.

THEOREM. Let $A$ be a closed subset of the closed unit disc $D^{-}$, such that $A \cap \partial D$ has (linear) Lebesgue measure zero. Then there exists a function $\varphi(r, A)$ satisfying (1) such that (2) holds for every inner function $q(z)$ with $\operatorname{Sp}(q) \subset A$.

We shall need an auxiliary result.

LEMMA. Let $p, q$ be two inner functions and assume that $1-M(p, r) \leqq \alpha^{2}$, $1-M(q, r) \leqq \beta^{2}, 0 \leqq \alpha, \beta<1$ (for some $\left.r, 0 \leqq r<1\right)$. Then $1-M(p q, r) \leqq$ $3(\alpha+\beta)$.

ProOf. Let $K(p, \alpha, r)=\left\{e^{i x} \in \partial D:\left|p\left(r e^{i x}\right)\right|>1-\alpha\right\}$ and let $K(q, \beta, r)$ be similarly defined. Since $|p(z)| \leqq 1$, the inequality $1-M(p, r) \leqq \alpha^{2}$ and a straightforward computation show that the measure of $K(p, \alpha, r)$ must be greater than $1-\alpha$. Similarly, $m(K(q, \beta, r))>1-\beta$ and therefore, if

$$
N(p q, r)=\left\{e^{i x} \in \partial D:\left|p\left(r e^{i x}\right) q\left(r e^{i x}\right)\right|>(1-\alpha)(1-\beta)\right\},
$$

then $m(N(p q, r))>(1-\alpha)(1-\beta)$ (observe that $N(p q, r)$ contains $K(p, \alpha, r) \cap$ $K(q, \beta, r))$, whence the result follows. Q.E.D.

Using this lemma, it will be enough to prove the Theorem for Blaschke products and for singular inner functions separately.

(a) Blaschke Products. Consider the function

$$
b(z, a)=(\bar{a} /|a|) \cdot(a-z) /(1-\bar{a} z), \quad a=r e^{i t} \in D,
$$


and let $\varepsilon, 0<\varepsilon<1$, be given; then the set $\{z:|b(z, a)|<1-\varepsilon(1-r)\}$ is a disc having the rectilinear segment $\left[-r_{i} e^{i t} ; r_{s} e^{i t}\right]$, where $r_{i}=(1-\varepsilon) /(1+\varepsilon r)$ and $r_{s}=(1-\varepsilon+r+\varepsilon r) /\left(1+r-\varepsilon r+\varepsilon r^{2}\right)$, as a diameter. We have $r_{i}<1-\varepsilon$, $r_{s}<1-\varepsilon(1-r)^{2}$.

Let $A$ be as in the Theorem and let $b(z)=\prod_{k} b\left(z, a_{k}\right)$ be a Blaschke product such that $a_{k} \in A \cap D$ for all $k$, and $\sum_{k}\left(1-\left|a_{k}\right|\right) \leqq M<\infty$. We are going to construct a function $\alpha(r, A, M)^{2}$ satisfying the conditions (1), such that $1-M(b, r) \leqq \alpha(r, A, M)^{2}$, for all Blaschke products satisfying the above conditions.

For each $\varepsilon, 0<\varepsilon<1$, define

$$
A_{\varepsilon}=\bigcup\{z:|b(z, a)|<1-\varepsilon(1-|a|) ; a \in A \cap D\},
$$

and let $D_{\varepsilon, t}$ be the disc having the rectilinear segment

$$
\left[-(1-\varepsilon) e^{i t} ;\left(1-\varepsilon\left(1-r_{t}\right)^{2}\right) e^{i t}\right],
$$

where $r_{t}=\max \left\{r: r e^{i t} \in A\right\}(0 \leqq t \leqq 2 \pi)$, as a diameter. The above estimate shows that

$$
A \cap D \subset A_{\varepsilon} \subset A_{\varepsilon}^{\sim}=\text { closure } \bigcup\left\{D_{\varepsilon, t}: 0 \leqq t \leqq 2 \pi\right\} .
$$

It follows that $A \subset \bigcap\left\{\left(A_{\varepsilon}^{\sim}: 0<\varepsilon<1\right\}\right.$ and $A \cap \partial D=A_{\varepsilon}^{\sim} \cap \partial D$, for all $\varepsilon$. Furthermore, if $A_{\varepsilon, r}=\left\{e^{i t}: r e^{i t} \in A_{\varepsilon}^{\sim}\right\}$, then

$$
\lim (r \rightarrow 1) m\left(A_{\varepsilon, r}\right) \leqq m(A \cap \partial D)=0 .
$$

Now, if $z \in D \backslash \tilde{A_{\varepsilon}}$, then

$$
|b(z)|=\prod_{k}\left|b\left(z, a_{k}\right)\right| \geqq \prod_{k}\left[1-\varepsilon\left(1-\left|a_{k}\right|\right)\right] \geqq C(\varepsilon, M),
$$

where $C(\varepsilon, M) \rightarrow 1$ as $\varepsilon \rightarrow 0$ for fixed $M$ (namely, if $A$ does not intersect the disc of radius $r_{0}>0$ about the origin, then $C(\varepsilon, M)$ can be taken equal to $\left.\exp \left\{-\varepsilon M / r_{0}\right\}\right)$.

Fix $\varepsilon$ and choose $r$ so that $m\left(A_{\varepsilon, r}\right)<\varepsilon$. Then

$$
M(b, r)=\int_{0}^{2 \pi}\left|b\left(r e^{i x}\right)\right|^{2} d m \geqq C(\varepsilon, M)^{2}(1-\varepsilon) \rightarrow 1, \quad \text { as } \varepsilon \rightarrow 0 .
$$

It is clear that the relation between $\varepsilon$ and $r$ can be inverted in order to obtain a function $\alpha(r, A, M)^{2}$, which depends only on $M$ and the shape of $A$, such that $1-M(b, r) \leqq \alpha(r, A, M)^{2} \rightarrow 0$, as $r \rightarrow 1$, independently of the distribution of the zeroes $\left\{a_{k}\right\}$ in $A$.

(b) Singular Functions. Now consider the function $s(z)=e(z, \mu)$, where the measure $\mu$ is supported by $A \cap \partial D$. If $z=r e^{i x}$, then

$$
|s(z)|=\exp \left\{-\int_{0}^{2 \pi} P(r, x-t) d \mu(t)\right\},
$$


where $P(r, x-t)=\left(1-r^{2}\right) /\left(1+r^{2}-2 r \cos (x-t)\right)$ is the Poisson kernel of the disc. This expression shows that the infimum of $|s(z)|$ over all functions $s(z)$ satisfying the above conditions and such that $\|\mu\| \leqq M$ is actually attained by taking $\mu=M \delta_{c(z)}$, where $\delta_{c(z)}$ is the Dirac measure placed at one of the points $c(z)$ such that

$$
|z-c(z)|=\min \left\{\left|z-e^{i t}\right|: e^{i t} \in A\right\} \quad(|c(z)|=1) .
$$

Now observe that the set $\{z:|d(z ; 0, M)| \leqq 1-\varepsilon\}$ (where $d(z ; t, N)=$ $\left.\exp \left\{N\left(z+e^{i t}\right) /\left(z-e^{i t}\right)\right\}\right)$ is the closed disc having the rectilinear segment $\left[-r_{\varepsilon, M} ; 1\right]$ where $r_{\varepsilon, M}^{2}=[M+\log (1-\varepsilon)] /[M-\log (1-\varepsilon)]$, as a diameter.

Let $D_{\varepsilon, M, t}$ be the closed disc having the rectilinear segment $\left[-r_{\varepsilon, M} e^{i t} ; e^{i t}\right]$ as a diameter, and define

$$
B_{\varepsilon, M}=\bigcup\left\{D_{\varepsilon, M, t}: e^{i t} \in A\right\} .
$$

We have $A \cap \partial D=\bigcap\left\{B_{\varepsilon, M}: 0<\varepsilon<1\right\}=B_{\varepsilon, M} \cap \partial D$ for all $\varepsilon$, and

$$
\lim (r \rightarrow 1) m\left(\left\{e^{i t}: r e^{i t} \in B_{\varepsilon, M}\right\}=m(A \cap \partial D)=0 .\right.
$$

Now we can proceed as in Case (a) to obtain a function $\beta(r, A, M)^{2}$ satisfying the conditions (1), which only depends on $M$ and the shape of $A$, such that $1-M(s, r) \leqq \beta(r, A, M)^{2}$, independently of the mass distribution of the measure $\mu$ on $A \cap \partial D$.

From (a), (b) and the Lemma, it follows that

$1-M(q, r)=1-M(b s, r) \leqq 3[\alpha(r, A, M)+\beta(r, A, M)]=\varphi(r, A, M)$,

for any inner function $q(z)$ such that $\operatorname{Sp}(q) \subset A$ and $\sum_{k}\left(1-\left|a_{k}\right|\right) \leqq M$, $\|\mu\| \leqq M$.

Finally, if $\varphi(r, A)$ is any function satisfying (1) such that

$$
\lim (r \rightarrow 1) \varphi(r, A, M) / \varphi(r, A)=0,
$$

for all $M>0$ (such a function can be easily constructed by standard arguments), then $1-M(q, r)=O(\varphi(r, A))$, for all $q(z)$ with $\operatorname{Sp}(q) \subset A$.

The proof of the Theorem is complete now.

EXAMPLE. Let $\varphi(r)$ satisfy (1). There exist a Blaschke product $b(z)$ and a sequence of radii $0<r_{1}<\cdots<r_{n}<\cdots<1$ converging to 1 , such that $1-M\left(b, r_{n}\right)>_{n} \cdot \varphi\left(r_{n}\right)$, for all $n$. Furthermore, $b(z)$ can be constructed so that $\operatorname{Sp}(b) \cap \partial D=\{1\}$.

The construction is simple. Let $A_{n}=\left\{e^{i t}:|t|<\pi / 2^{n}\right\}$, and let

$$
f_{n}(z)=\exp \left\{-\int_{-\pi / 2^{n}}^{\pi / 2^{n}}\left(e^{i t}+z\right) /\left(e^{i t}-z\right) d m(t)\right\} .
$$


Then $\left|f_{n}(z)\right|<1$ on $D$ and

$$
\begin{aligned}
\left|f_{n}\left(e^{i x}\right)\right| & =e^{-1} & & \text { if } e^{i x} \in A_{n} \\
& =1 & & \text { if } e^{i x} \in \partial D \backslash A_{n}
\end{aligned}
$$

Construct the sequence $\left\{r_{n}\right\}$ by induction, so that $n \cdot \varphi\left(r_{n}\right)<2^{-n-4}$. Let $-\pi / 2^{n}=y_{0}<y_{1}<\cdots<y_{N}=\pi / 2^{n}$ be a sufficiently small partition of $A_{n}$ so that $y_{j}-y_{j-1}=2 \pi / 2^{n} N, j=1, \cdots, N$, and

$$
\left|f_{n}(z)-\prod_{j=1}^{N} d\left(z ; y_{j}, 1 / 2^{n} N\right)\right|<4^{-n-5}, \text { for }|z| \leqq r_{n} .
$$

Approximate each of the functions $d\left(z ; y_{j}, 1 / 2^{n} N\right)$ by a Blaschke product whose zeros cluster at $e^{i y_{j}}$ (this can be done by using a classical result of O. Frostman, see [2]) and then approximate each of these Blaschke products by a suitable partial finite product on the closed disc of radius $r_{n}$. Thus we can obtain a finite Blaschke product $b_{n}(z)$ such that

(1) $\left|f_{n}(z)-b_{n}(z)\right|<4^{-n-5}$, for $|z| \leqq r_{n}$, and

(2) $b_{n}\left(r e^{i x}\right) \neq 0$, if $r<r_{n}$ or $e^{i x} \notin A_{n-1}$.

Define $b(z)=\prod_{n=1}^{\infty} b_{n}(z)$; we have

$$
b(0)=\prod_{n} b_{n}(0)=\prod_{n} f_{n}(0)\left[b_{n}(0) / f_{n}(0)\right]>e^{-1} \prod_{n}\left(1-4^{-n-5}\right)>0 \text {. }
$$

Therefore, $b(z)$ is actually a Blaschke product (see [2]), and

$$
\begin{aligned}
1-M\left(b, r_{n}\right) & \geqq 1-M\left(b_{n}, r_{n}\right) \\
& \geqq 1-M\left(f_{n}, r_{n}\right)+\left[M\left(f_{n}, r_{n}\right)-M\left(b_{n}, r_{n}\right)\right] \\
& >1-M\left(f_{n}\right)-2^{-n-4}>2^{-n-3}-2^{-n-4}>n \cdot \varphi\left(r_{n}\right) .
\end{aligned}
$$

It is apparent that $z=1$ is the only cluster point for the zeroes of $b(z)$. It is also clear that a similar method can be used to prove that, if $C \subset \partial D$ is any closed subset of positive Lebesgue measure, then there exists a singular inner function $s(z)=e(z, \mu)$ such that $\operatorname{supp}(\mu) \subset C$ and, for a suitable increasing sequence of radii converging to $1,1-M\left(s, r_{n}\right)>$ $n \cdot \varphi\left(r_{n}\right)$, for all $n$.

A Final Remark. The referee called our attention to the following result: If $s(z)$ is a (nonconstant) singular inner function, then $1-M(s, r)$ cannot converge to zero "too fast" (as $r \rightarrow 1$ ). In fact, it was shown in [4] that $s(z)$ is subordinated to $d(z ; 0, R)$ (for some $R>0$ ); now we can apply the corollary on p. 165 of [3] to show that $M(s, r) \leqq M(d, r)$, from which we can deduce that

$$
\left.1-M(s, r) \geqq 1-M(d, r) \geqq c(1-r)^{1 / 2} \quad \text { (for some } c>0\right) ;
$$

i.e.,

$$
1-M(s, r) \neq o\left((1-r)^{1 / 2}\right)
$$




\section{REFERENCES}

1. D. A. Herrero, Inner function-operators, Dissertation, University of Chicago, Chicago, Ill., 1970.

2. K. Hoffman, Banach spaces of analytic functions, Prentice-Hall Series in Modern Analysis, Prentice-Hall, Englewood Cliffs, N.J., 1962. MR 24 \#A2844.

3. J. E. Littlewood, Lectures on the theory of functions, Oxford Univ. Press, Oxford, 1944. MR 6, 261.

4. D. J. Newman and H. S. Shapiro, The Taylor coefficients of inner functions, Michigan Math. J. 9 (1962), 249-255. MR 26 \#6371.

Department of Mathematics, University of Chicago, Chicago, Illinois 60637

Department of Mathematics, State University of New York, Albany, New YORK 12222

Current address: Departamento de Matématica, Universidade Estadual de Campinas, Caixa Postal 1170, 13100 Campinas, São Paulo, Brasil 